\title{
Retrospective Denial as A Coping Method
}

\author{
Ester J. M. Siemerink • Jan P. C. Jaspers • \\ John T. M. Plukker • Nanno H. Mulder • \\ Geke A. P. Hospers
}

Published online: 26 February 2011

(C) The Author(s) 2011. This article is published with open access at Springerlink.com

\begin{abstract}
Worldwide, gastric cancer is one of the most common and fatal cancers. The majority of patients present with an advanced stage of disease. Even with use of palliative chemotherapy most patients die within 1 year after diagnosis. Medical psychological attention after a diagnosis of incurable cancer is focused on end of life support. This paper presents the care of a patient treated with palliative intent with chemotherapy for an irresectable histologically confirmed gastric cancer. When, unexpectedly prolonged symptom free survival followed, the reaction of the patient came as a surprise to the attending medical team. In this case history we urge those who care for incurable cancer patients, that the rare patient who survives against all odds may require special psychological care.
\end{abstract}

Keywords Gastric cancer · Chemotherapy · Denial · Coping methods

E. J. M. Siemerink · N. H. Mulder $(\bowtie)$ - G. A. P. Hospers Department of Medical Oncology, University Medical Center Groningen, University of Groningen, 30.001, 9700 RB Groningen, The Netherlands

e-mail: n.h.mulder@int.umcg.nl

J. P. C. Jaspers

Department of Psychology, University Medical Center Groningen, University of Groningen, 30.001, 9700 RB

Groningen, The Netherlands

J. T. M. Plukker

Department of Surgery, University Medical Center Groningen, University of Groningen, 30.001, 9700 RB Groningen, The Netherlands

\section{Background}

Gastric cancer is one of the most common and fatal cancers, affecting more males than females. Large differences exist in the incidence of gastric cancers between various geographic regions. In North America, Africa, Oceania 4-10/100,000 people per year are affected, in northeast Asia up to 69 cases per 100,000 people per year are affected (Hartgerink, Jansen, van Grieken \& van de Velde, 2009).

It is now known that this cancer is generally the aftermath of Helicobacter Pylori (H. Pylori) infection. The risk for adenocarcinoma is 6-fold increased in those infected with H. Pylori. H. Pylori is a bacterium which can lead to chronic gastritis. However only a minority of patients infected with this organism develop cancer, concerting risk factors are high salt intake, low intake of vegetables or fruit, obesity and smoking (Helicobacter Cancer Collaborative Group 2001). There are two distinct histological types of gastric adenocarcinoma. The intestinal type of gastric cancer affects more males and older age groups. The diffuse type of gastric cancer is equally frequent in both sexes and more common in younger age groups, and has a worse prognosis than the intestinal type.

Although the incidence of gastric cancer is declining due to preventive strategies, such as treatment of $\mathrm{H}$. Pylori and better food preservation methods, it remains the second leading cause of cancer related death worldwide. Symptomatic patients often have complaints like weight loss, abdominal pain or dysphagia. Diagnosis is established by endoscopy, which is insertion of a flexible tube through the mouth (Hartgerink et al., 2009). Once the diagnosis is established by obtaining tissue scored by the pathologist as being malignant, therapy can be aimed at cure or be limited to some prolongation of life and mitigation of symptoms. 
The only way to cure gastric cancer is by surgery. Because of the high recurrence rate after surgery alone most patients are also treated with some form of adjuvant (postoperative) or neoadjuvant (preoperative) chemotherapy, in the United States combined with radiotherapy. If surgery can be done pre-operative measures require CT scanning and often ultrasound (Hartgerink et al., 2009). Sometimes it is only during surgery that it becomes clear that the cancer has progressed beyond the surgeon's domain. In such advanced stages of gastric cancer, when the tumor has become irresectable, attention has to be focused on palliative measures. The emphasis in this phase is based on prolonging survival with preservation or improving quality of life. Care is then largely determined by the expectancy of approaching death. Although still a demanding clinical task, much literature and guidance is available for the clinician on giving optimal support. Compared to best supportive care, several studies have described a beneficial effect of chemotherapy on quality of life, next to achievement of survival benefit. Compared to mono chemotherapy agents, combination chemotherapy gives additional survival benefit of some weeks to months, at the expense of more toxicity (Wagner et al., 2006). With the development of targeted agents against molecular signaling pathways a larger survival benefit might be accomplished. Targeted therapy is a type of medication which blocks the growth of cancer cells by interfering with specific targeted molecules needed for tumor growth rather than interfering with rapidly dividing cells in the case of traditional chemotherapy (Hartgerink et al., 2009).

Next to chemotherapy or local palliative measurements physicians get trained in guiding patients on their way to approaching death, by listening to their complaints, desires and fears. Surprisingly little is known about the psychological consequences for the rare patient with unexpected long-term survival or cure against all odds, as described here.

\section{Case Report}

A middle-aged married woman was referred after explorative laparotomy because of irresectable intestinal type adenocarcinoma of the stomach. Until then she had an active social life, participated in labor with pleasure and enjoyed spending time with her grandchildren. Chemotherapy was instituted, but stopped because of excessive toxicity. The message communicated to her and her husband at that moment was gloomy based on the initial diagnosis and the residual lesions on CT-scan after chemotherapy. Even more than before the start of chemotherapy, the patient at the age of 61 expected to die from this cancer and seemed to adapt to this situation, especially since a second opinion, confirmed the initial diagnosis and did not provide alternatives. However the patient remained in an excellent condition throughout the following years.

Although one could assume that a patient would be relieved by being an exceptional long-term survivor, 7 years by now, the opposite occurred. Survival became a considerable psychological problem. Finally some form of equilibrium was found in a state of denial of ever having had cancer.

\section{Discussion}

The median survival of patients with advanced gastric cancer is $7-10$ months. Chemotherapy can lead to palliation and prolongs survival with a few months. However long-term survival or cure after only a partial tumor remission on chemotherapy without surgery has rarely been described (Wagner et al., 2006).

Apart from these medical considerations the most remarkable reaction of our patient was the psychological one. At first the patient accepted the diagnosis of irresectable gastric cancer and agreed to palliative chemotherapy. However, after remaining alive considerably longer than expected without evidence of disease our patient reacted with a rare type of denial namely retrospective denial of the diagnosis.

Denial in cancer patients is a well recognized phenomenon in clinical practice (Heim, Augustiny, Schaffner, \& Valach, 1993; Rabinowitz \& Peirson, 2006; Sharf, Stelljes, \& Gordon, 2005; Vos \& de Haes, 2007). Denial is the process by which the mind defends itself against painful or threatening information. Depending on the situation this can be maladaptive, e.g., leading to treatment delay, or adaptive, e.g., enabling the patient to cope with this lifethreatening disease. A recent review on denial in cancer patients distinguished four types of denial: denial of diagnosis, denial of impact, denial of affect, and behavioral escape (Helicobacter Cancer Collaborative Group 2001). Denial of diagnosis mostly takes place early in the process, and decreases over time, although it sometimes increases during the terminal phase (Heim et al., 1993).

Our patient, however, seems to be cured and denying having had cancer at first sight seems incomprehensible. Insight in this denial can be obtained from available knowledge on how people try to explain unpredictable life events. In general, people make different causal attributions for positive and negative events. Positive events mostly are considered to be the result of internal causes: people tend to ascribe success in life to themselves. Negative events are mostly attributed to external causes: failure due to circumstances. So, we could expect that our patient would attribute being a survivor of cancer to these internal 
causes, such as a fighting spirit, or a healthy life style. Most patients will make causal attributions to external sources for the fact that they got cancer, clearly a negative event, in such a way that self-esteem is preserved (Sharf et al., 2005).

When our patient stayed alive longer than expected external, rather than internal attributions were sought by the patient. The patient appeared to begin to believe that given the fact that the doctors were wrong about dying soon they could be wrong in other aspects of the disease too. If the patient did not believe in divine intervention, how could this unexpected outcome be explained? The thought of not having cancer in the first place is in accordance with this unexpected outcome. Moreover, this conviction serves well to reduce anxiety and uncertainty about recurrence of cancer: a predominant preoccupation in most survivors of cancer (Rabinowitz \& Peirson, 2006; Vos \& de Haes, 2007). There was no reason to be afraid that the cancer could come back if it had never been there! Although denial in this case is not a logical solution, it certainly is a psychological one. Thus for the patient the past experiences with life-threatening disease and the unexpected positive outcome are explained, and the fear of a recurrence of cancer is superfluous.

As a medical team we were unable to help the patient on the path from the certainty of death to unexpected survival. Whether the adaptation through denial of the patient to the new situation is the most desirable one is doubtful. Realization by us that good news may be as difficult to cope with as bad news might have provided a guideline for earlier psychological intervention.

Dialogue between Drs. Siemerink (junior Medical Oncologist), Dr. Jaspers (Psychologist) and Professor Mulder (senior Medical Oncologist) regarding the case:

Drs. Siemerink: Professor Mulder, how often have you observe unexpected prolonged survival among patients with advanced disease? And is there in your opinion a common reaction in your patients to that situation?

Professor Mulder: There are various levels of unexpected survival, most often reported in the context of some form of alternative medicine. In a review in our country some 400 reports of that kind were analyzed and only 2 could be accepted as undoubtful. If we give a prognosis of 8-12 months for a patient as described here we would expect an occasional patient to live for 2 years, just as some patients with breast cancer may survive for decades with metastatic disease. However for a patient treated in a palliative context to be cured, as in this situation, I can remember three such cases in a period of some 30 years, all of them were extremely grateful, if not to me at least to their good fortune.

Professor Mulder: Dr. Jaspers, the reaction of our patient to survival, retrospective denial of the diagnosis of cancer, came as a complete surprise for the medical team. Is this reaction common in cancer patients?

Dr. Jaspers: Denial in cancer patients is a well recognized phenomenon in clinical practice, especially denial of diagnosis. However, it mostly takes place early in the process of diagnosis, and decreases over time. Sometimes it increases during the terminal phase. So, denial is a common reaction to bad news. What makes denial exceptional in this case, is that it is a reaction to the good news of survival. Other causal attributions for this positive event could be expected. People normally attribute positive events to internal causes, in the case of cancer survival for instance to their fighting spirit or to their change of lifestyle. When a patient attributes his survival to his own behavior or characteristics, there is no need to deny the fact that he had cancer.

Drs. Siemerink: At first we did not understand the reaction of the patient who was very angry at us and was convinced that we had failed as doctors and caused much sorrow.

Dr. Jaspers: Indeed, at first sight it seems incomprehensible that a positive progress of the disease made the patient react this way. But from the point of view of the patient, if it would be true that cancer had never been there the emotions can be understood. In the patient's mind, the patient was told that this disease was fatal and treated invasively for a disease which was not there.

Drs. Siemerink: So, the retrospective denial of the diagnosis is a way of coping with this unexpected positive course of the disease?

Dr. Jaspers: Cancer is a disease that brings much uncertainty and loss of control for patients. In general there is little they can do, except rely on medical treatment and hope for the best. Loss of control is hard to deal with, and research on coping with cancer shows that at least half of the surviving patients experience positive effects (posttraumatic growth) of having cancer. Some consider these positive effects as a way of coping to regulate feelings of anxiety and depression (illusionary growth), others consider these positive experiences as real growth (Zoellner \& Maercker, 2006). Retrospective denial of the diagnosis is an effective way to regain control and at the same time eliminate anxiety and uncertainty about recurrence of cancer.

Professor Mulder: At the other hand, denial as a way of coping seems unrealistic. Can denial be an effective coping method?

Dr. Jaspers: Denial is the process by which the mind defends itself against painful or threatening information. Mostly denial does not last long, because it is only temporarily necessary to regulate and control negative emotions. In the long run denial is often inadequate, because it hampers adaptation to the altered situation, for instance 
having cancer. So, it depends on the circumstances whether denial can be an adequate or inadequate coping strategy. In the case of this patient denial not only explains the unexpected cure of cancer, but also seems to have positive effects on the long run (reduce anxiety about recurrence of cancer). So, may be in this case denial is not a bad strategy for the patient as long as this strategy has no evident disadvantages for the patient. There may be another reason for this unexpected attribution. In general, people have a strong urge to seek causal attributions for their behavior or for the events they experience. The cure of irresectable gastric cancer cannot be explained. It is hard for people to live with such events of vital importance that cannot be explained. So, retrospective denial of the diagnosis makes sense, because it explains the inexplicable: it is better to have an improbable explanation than no explanation at all.

Drs. Siemerink: What can we learn from this case? The reaction of the patient resulted in premature loss of contact with the patient. We realized too late that the good news was difficult to cope with.

Dr. Jaspers: Indeed, the first lesson to learn from this case is that good news may be as difficult to cope with as bad news. To realize this and to speak with the patient about this unexpected progress and the possible confusing impact of it can be the first step. To share feelings with the patient over this positive, but unlikely development can give the physician the opportunity to consider whether psychological intervention is indicated. Doubts about the diagnosis can be discussed earlier by the patient when he feels he is free to do so without direct rejection of this possibility. I think, physicians should respect this opinion of the patient, baring in mind that this attribution has a psychological function. Direct attempts to refute this opinion only increases resistance to chance or reconsideration of this opinion and hinders discussion of other issues for example how to adapt to the fact that life is not over yet and how the patient can plan for the future again. Perhaps, for some physicians the conviction of the patient that the diagnosis was wrong is a difficult one, because their expertise is called in question. Of course, the patient has the right to be informed about all aspects of his disease, but sometimes the truth of the medical team is not the truth of the patient.

Dr. Jaspers: how are you as a young oncologist trained in dealing with these difficult situations of providing information about the fatal course of a disease in general but also staying focused on the individual patient?

Drs. Siemerink: In our training much attention is paid to communication of bad news and the different ways a patient can react to this news at diagnosis, for example by patient simulation and watching videos. However this specific situation of sustained denial was not discussed in my training in its place.
Professor Mulder: If we had recognized the problem earlier, and had consulted a psychologist, would he have tried to reverse this process? In other words, given that the patient has reached some form of equilibrium, can we consider this to be a satisfactory situation? Not quite unconnected is the question if it is likely that at some time the patient will realize that cancer indeed had occurred.

Dr. Jaspers: I don't think the reason for consulting a psychologist should be to convince the patient of the correctness of the diagnosis of cancer, but to explore with the patient what contrary to all expectations, this unexpected survival means for the patient. I think it is important for the patient to express (mixed) emotions and to talk about the reactions to this confusing course of the disease. Undoubtedly, in talking about the situation the question will arise: 'How can this be?' It is hard to speculate whether this patient would have considered other answers than the conviction that cancer never had occurred, but I suppose that earlier in the process this idea is still more open for discussion. When the patient is still looking for an answer, the psychologist can explain that it is our human nature to attribute causes to all important events that we experience, also the events that perhaps are inexplicable. So, it may be difficult to realize that certainty about the cause of progress of the patient's disease cannot be found. But, if the patient is already convinced that the doctor was wrong in his diagnosis, I think it would be wise to respect this opinion, given the psychological function that we discussed before. This does not mean the psychologist has to agree with the patient: he can state that-just like the medical team-he cannot give an explanation for this unexpected development. What the psychologist can do for the patient is to sort out how to adapt to this new situation, prevent obsessive preoccupation and rumination, and how to get on with life, leaving this stressful period of illness behind. When the patient succeeds in this respect, the urgency to blame the physicians for the alleged faults they made will be less, and the conclusion that the patient was very lucky in the end can be acceptable.

Drs. Siemerink: Finally a question that, perhaps, cannot be answered: should this extraordinary case change the information we give an incurable patient or if there is no statistical hope should we still provide it?

Dr. Jaspers: In the last decades there has been a major change in the way we talk about cancer and in our view how to inform the patient. Although the patient has the (legal) right to be informed fully, sometimes the truth and nothing but the truth can be too much to handle for the patient. Appraisal of the state of mind of the patient can be difficult and to bring the bad news of approaching death remains a demanding clinical task, but in general the information available should be given. Timing, choosing the right words and opportunity are important in telling the 
unwelcome truth, but this truth gives the patient and his beloved ones the opportunity to deal with it. So, when there is no (statistical) hope, no false hope should be given. When the patient needs hope to endure his situation, he will find it anyway, holding back the truth is not a good way to provide hope.

\section{Conflict of interest None.}

Open Access This article is distributed under the terms of the Creative Commons Attribution Noncommercial License which permits any noncommercial use, distribution, and reproduction in any medium, provided the original author(s) and source are credited.

\section{References}

Hartgerink, H. H., Jansen, E. P., van Grieken, N. C., \& van de Velde, C. J. (2009). Gastric cancer. Lancet, 374, 477-490.
Heim, E., Augustiny, K. F., Schaffner, L., \& Valach, L. (1993). Coping with breast cancer over time and situation. Journal of Psychosomatic Research, 37, 523-542.

Helicobacter Cancer Collaborative Group. (2001). Gastric cancer and Helicobacter pylori: A combined analysis of 12 case control studies nested within prospective cohorts. Gut, 49, 347-353.

Rabinowitz, T., \& Peirson, R. (2006). "Nothing is wrong, doctor": Understanding and managing denial in patients with cancer. Cancer Investigation, 24, 68-76.

Sharf, B. F., Stelljes, L. A., \& Gordon, H. S. (2005). 'A little bitty spot and I'm a big man': Patients' perspectives on refusing diagnosis or treatment for lung cancer. Psychooncology, 14, 636-646.

Vos, M. S., \& de Haes, J. C. (2007). Denial in cancer patients, an explorative review. Psychooncology, 16, 12-25.

Wagner, A. D., Grothe, W., Haerting, J., Kleber, G., Grothey, A., \& Fleig, W. E. (2006). Chemotherapy in advanced gastric cancer: A systematic review and meta-analysis based on aggregate data. Journal of Clinical Oncology, 24, 2903-2909.

Zoellner, T., \& Maercker, A. (2006). Posttraumatic growth in clinical psychology-A critical review and introduction of a two component model. Clinical Psychology Review, 26, 626-653. 